\title{
Tissue Engineering and Regenerative Medicine, From and Beyond the Dentistry
}

\author{
Wenkai Jiang ${ }^{1,2}$, Longxing $\mathrm{Ni}^{2}$, Alastair Sloan ${ }^{1}$ and Bing Song ${ }^{1,2 *}$
}

${ }^{1}$ School of Dentistry, Cardiff Institute of Tissue Engineering and Repair, Cardiff University, Heath Park, Cardiff, CF14 4XY, UK

${ }^{2}$ State Key Laboratory of Military Stomatology, Department of Operative Dentistry and Endodontics, School of Stomatology, the Fourth Military Medical University,

710032, Shaanxi, P R China

\begin{abstract}
In the past decade, oral stem/progenitor cells have been proved to be a promising source for tissue engineering and regenerative medicine, due to their multiple advantages of easy accessibility without invasive procedures and ethical issues, excellent proliferation and regeneration capacity, pluripotency of differentiation into multiple lineages, as well as little inherent immunogenicity. There are a variety of oral tissue derived stem/progenitor cells, including dental pulp stem cells (DPSCs), stem cells from exfoliated deciduous teeth/apical papilla/periodontal ligament, mesenchymal stem cells from gingiva, and progenitor cells from oral mucosal lamina propria (OMLP-PCs). This review outlines two major oral stem/progenitor cells - DPSCs and OMLP-PCs, and showcases their potential in not only regenerative dentistry but also regenerative treatments for other systems and immunotherapies.
\end{abstract}

Keywords: Dental pulp stem cells; Periodontal ligament stem cells; Progenitor cells

\section{Introduction}

To restore the damaged tissue or organs, it is critical to understand the developmental process behind the tissue engineering and regenerative medicine. Stem cells play essential roles in tissue engineering for organ development and tissue repair. Two main types of stem cells have been studied including embryonic stem cells (ES cells) and postnatal or adult stem cells (AS cells). ES cells derived from the blastocyst have the ability to differentiate into multipotent stem / progenitor cells, including epithelial, mesenchymal, and other tissuespecific stem cells $[1,2]$. The specialized functional tissue and organs of human body can be generated by the interactions among these stem cells $[3,4]$. Once the organ matures, the pluripotent ES cells evanesce. Some multipotent AS cells however remain in the developed tissue and have the ability to regenerate and/or repair injuries [5], for example mesenchymal stem cells (MSCs) derived from bone marrow [6], dental pulp [7], adipose tissue [8], dermis [9] and umbilical cord [10].

Oral stem cells mainly include dental pulp stem cells (DPSCs) [7], stem cells from exfoliated deciduous teeth (SHED) [11], stemcells from apical papilla (SCAP) [12], periodontal ligament stem cells (PDLSCs) [13], mesenchymal stemcells from gingiva (GMSCs) [14] and progenitor cells from oral mucosal lamina propria (OMLP-PCs) [15]. They are widely applied in not only regenerative dentistry but also tissue and organ repair and/or regeneration of other systems.

We showcase in this mini-review the past development and current status of two representative oral stem/progenitor cells: DPSCs and OMLP-PCs, and their prospective applications in tissue engineering and regenerative medicine.

\section{Dental Pulp Stem Cells}

DPSCs are mesenchymal-derived cells residing within the perivascular niche of dental pulp and are considered to originate from migrating cranial neural crest cells. These cells are multipotent stem cells which have advantages for clinical applications [16]. Compared with other MSCs derived from bone marrow, adipose tissue, peripheral blood, and umbilical cord blood, DPSCs has a distinct advantage of easy accessibility with entirely non-invasive procedures and little ethical issues. This is because healthy adult teeth, particularly impacted molars are often extracted during orthodontic treatment. Rather than discarding the teeth, pulp tissue can be excavated and cryogenically preserved. DPSCs can subsequently be isolated from such tissue, following thawing, with no detrimental effects in viability, proliferation or differentiation [17]. This has led to the establishment of pulp tissue banks for patients to store and use their own progenitor cell samples if and when required. A number of studies also demonstrated that the immunogenic reaction to DPSCs may be suppressed by the inhibited activation and induced apoptosis of T-cells [18-20]. This suggests that in addition to patient-matched autologous applications, DPSCs may be tolerated as an allogeneic cell transplant without the need for immunosupression [21].

The presence of DPSCs was first proposed by Gronthos et al. [7]. DPSCs has been demonstrated to express a range of mesenchymal stem cells related markers such as CD44, CD73, CD90, CD105, stem cell antigen (SCA1) and stro-1 [22], therefore they are generally classified as mesenchymal derived stem cells. DPSCs can be readily induced to differentiate in-vitro into the classic MSC lineage cell types of osteoblasts/odontoblasts, chondrocytes and adipocytes using established protocol $[23,24]$. However, the differentiation potential of DPSCs may not be strictly limited to mesenchymal cell types, since the expression of pluripotency markers such as nanog, oct 4 , sox 2 and ssea 4 can be found in cultured DPSCs [25]. Additionally, DPSCs have been demonstrated to express markers related to tissues derived from other germ layers such as muscle ( $\alpha$-smooth muscle actin) [22] and neurons (Nestin, BIII-tubulin and Map2) [25]. A number of studies have demonstrated the potential for endothelial [26], myogenic [27], hepatocytic [28] and melanocytic differentiation [29]. Due to this potential versatility, a variety range of therapeutic applications have been proposed for DPSCs. In-vivo, a number of DPSCs based studies

*Corresponding author: $\mathrm{Dr}$. Bing Song, Tissue Engineering and Regenerative Dentistry, School of Dentistry, Cardiff University, Heath Park, Cardiff, CF14 4XY UK, Tel: +44 29207 44182; E-mail: SongB3@cardiff.ac.uk; JiangW6@cardiff.ac.uk

Received April 06, 2015; Accepted May 13, 2015; Published May 17, 2015

Citation: Jiang W, Ni L, Sloan A, Song B (2015) Tissue Engineering and Regenerative Medicine, From and Beyond the Dentistry. Dentistry 5: 306. doi:10.4172/2161-1122.1000306

Copyright: $\odot 2015$ Jiang W, et al. This is an open-access article distributed under the terms of the Creative Commons Attribution License, which permits unrestricted use, distribution, and reproduction in any medium, provided the original author and source are credited. 
have been conducted to repair or regenerate non-dental tissues. De Mendonca et al. and Riccio et al. investigated that new bone could be formed in critical-size calvarial bone defects [30,31], while Yamada et al. and Zheng et al. found that critical-size mandibular bone defect could be repaired using DPSCs [32,33]. Ito et al. compared the osteogenic potential of three types of cells-DPSCs, bone marrow stem cells (BMSC) and periosteal cells (PC), for the osseointegration of dental implants and tissue engineered bone by transplanting these cells into the alveolar bone defect of dogs. Interestingly, the osteogenic ability of DPSCs was shown to be more superior than that of bone marrow-derived stem cells, indicating that DPSC was a useful cell source for tissue-engineered bone in dental implants [34]. Additionally, some of studies demonstrated that DPSCs play a potential role in the nerve repair and/or regeneration. DPSCs formed blood vessels and myelinating tissue and contributed to the promotion of peripheral nerve regeneration when transplanted into a gap in the rat facial nerve [35]. They could also induce neuroplasticity within a recipient host nervous system by secreting factors that coordinate axon guidance [36]. Apart from above, DPSCs also have positive effects in the modulation and enhancement of adult neurogenesis. The co-culture with DPSCs significantly protected primary cultures of hippocampal and ventral mesencephalic neurons in-vitro models of Alzheimer's and Parkinson's disease [37,38]. Additionally, DPSCs exert protective effect on dopamine (DA) neurons by increasing number of spared tyrosine hydroxylase $(\mathrm{TH})^{+}$cells [38]. These all proved that DPSCs can be deemed as possible candidates for cell-based therapy in adult neurodegenerative disorders in future. DPSCs have also demonstrated its potential in the Spinal Cord Injury (SCI) repair, which promoted the recovery of hind limb locomotor function in rats by inhibiting the SCI-induced apoptosis of neurons/astrocytes/oligodendrocytes and multiple axon growth inhibitors, and by replacing lost cells through differentiating into mature oligodendrocytes [39]. Furthermore, DPSCs have been proved to be a new stem cell source for cell-based therapy to stimulate angiogenesis/vasculogenesis during tissue regeneration. The cells have been shown to secrete new vasculature and express several proangiogenic factor, such as VEGF-A, G-CSF, GM-CSF, and MMP3 when transplanted into the model of mouse hind limb ischemia [26], and exhibited therapeutic potential for the repair of Myocardial Infarctions (MI) in a MI mouse model [40]. The cells from human deciduous teeth were also reported to accelerate wound healing in combination with basic fibroblast growth factor (b-FGF) [41].

\section{OMLP-PCs}

Oral Mucosal Lamina Propria progenitor Cells (OMLP-PCs) are progenitor cells of neural crest origin isolated from oral mucosal lamina propria (OMLP) [42] (Figure 1). OMLP-PCs which were first proposed by Stephens et al. have been demonstrated to express a range of stem cell markers including CD44, CD90, CD105 and CD166 [15]. These cells are generally classified as neural crest derived stem cells due to the present of Jagged 1 and the expression of a number of neural crest markers, such as Snail, Slug, Sox10 and Twist [15]. These progenitor cells have demonstrated their multipotency in-vitro

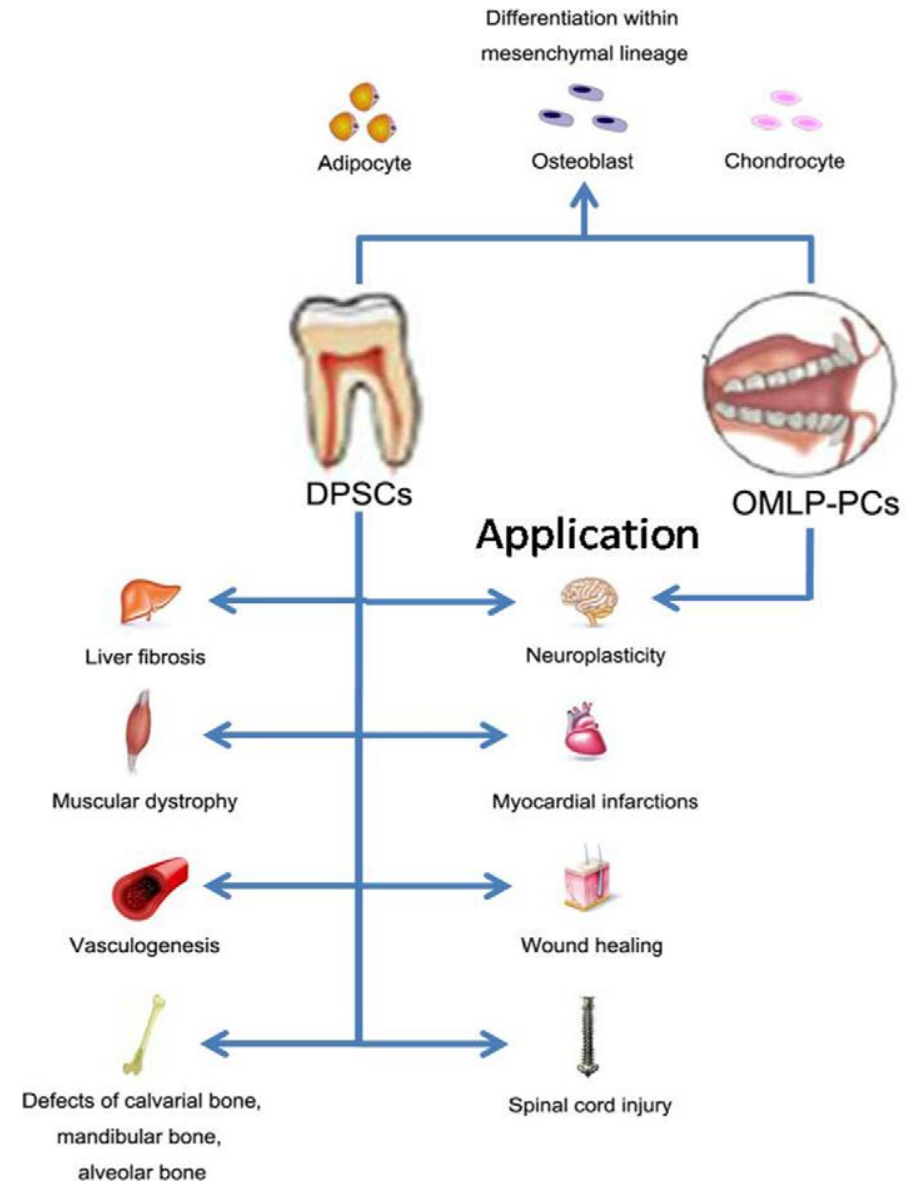

Figure 1: Multilineage differentiation capacity and potential clinical applications of dental pulp stem cells and oral mucosal lamina propriaprogenitor cells. 
with induced differentiation into both mesenchymal (chondrogenic, osteoblastic, and adipogenic) and neuronal (neuron and Schwann-like cells) cell lineages [15]. Additionally, the expression of pluripotency markers such as nanog, oct 4 and sox 2 can be found in cultured cells, which may indicate that the differentiation potential of OMLP-PCs may not be strictly limited to mesenchymal and neuronal cell types but can also be induced into other cell types derived from other germ layers. Therefore the potential differentiation of these progenitor cells offers a new approach for allogeneic tissue engineering application. Compared with other current stem cells sources, OMLP-PCs offers distinct advantages including easy accessibility, and minimally invasive isolation from the buccal mucosa, which heals without scar formation. Most importantly, OMLP-PCs has shown no inherent immunogenicity with minimal expression of costimulatory molecules (CD40, CD80, CD86, CD154 and CD178) or human leukocyte antigen (HLA) class II [43]. They can efficiently suppress the proliferation of peripheral blood lymphocytes in a dose dependent manner [43]. Compared with other mesenchymal stem cells, these characteristics make OMLP-PCs more advantaged for allogeneic tissue engineering as well as in the treatment of immunological disorders such as chronic graft-versus-host disease [43].

\section{Conclusion and Perspectives}

The rapid expansion of the current research on oral stem/ progenitor cells has shown encouraging outcome in tissue engineering and preclinical investigation. Stem cells from oral epithlium and mesenchymal tissue with advantages of easy accessibility and noninvasive extraction procedures offered minimal ethical issues in clinical application. Oral stem/progenitor cells demonstrated high proliferation rate, excellent regeneration capacity, multipotential of differentiation as well as little inherent immunogenicity, which guarantees their future clinical application potential in not only dentistry but also various other fields of regenerative medicine. However, several major objectives must be addressed before clinical application to be conducted, as below:

(1) Oral stem/progenitor cells have been demonstrated to be a promising cell source for the treatment of various pathological conditions, however the long-term fate of transplanted oral stem/ progenitor cells needs to be systematically clarified in the in-vivo models. It has been reported that in the spinal cord injury repair model, that massive cell death occurred at the grafting site with only a few transplanted cells survived, and most of them could not integrate into the local tissue [39]. The successful transition from in-vitro/ex-vivo to in-vivo is one of the key factors determining the efficiency of cell based therapy, in particular the standardized verification of the data from invitro/ex-vivo models in the in-vivo situation where cells respond to the endogenous environment largely differently compared to the in-vitro/ ex-vivo conditions. Under in-vitro/ex-vivo situation, the multipotency and differentiation capacity of stem / progenitor cells could be very well controlled with the aim of added growth factors and etc in the artificial culture system, which is very difficult to achieve once the cells are transplanted in-vivo. The multipotent differentiation capacity of oral stem/progenitor cells in-vitro does not necessarily reflect the response in-vivo, therefore the homing and transdifferentiation capacity and efficiency of oral stem/progenitor cells into a specific tissue type deserves more attention for investigation. Many pre-clinical/ translational studies reported encouraging outcome of the treatments, however some of these claims were based on marginal improvement (even though statistically meaningful), which might not be sufficient enough in the real clinical combat. Therefore, more stricter quantitative analysis should be put in place to evaluate the real therapeutic effect of these transplanted cells for tissue regeneration and injury repair.
(2) There is still an intense debate on the neuronal/glial differentiation of MSCs-like cells. An increasing number of studies have demonstrated that DPSCs have potential of neuronal/glial differentiation both in-vivo or in-vitro. In-vitro, multi-step pharmacological transdifferentiation protocols are proved to be efficient than simple differentiation procedures. These studies unequivocally showed neuronal morphological differentiation, and increased expression of neuronal/ glial markers at both gene and protein levels [24,44-49]. Additionally, some of the studies even provide evidence for the presence of at least some functional elements that are necessary for neuronal behaviour, such as specific calcium, sodium, and potassium channels $[44,46]$. However, the functional activation of these channels showed great variability depending on the different protocols of differentiation. Most of protocols could only induce one or two functional channels [44], therefore, most of DPSCs just differentiate into the neuronal/glial like cells in-vitro. Few of them demonstrate functional differentiation. Invivo, DPSCs have been used to transplant into a brain injury or a SCI in animal model $[39,50]$. Although DPSCs transplantation therapy could promote recovery of neuronal functions, little is known about the mechanism of how DPSCs work for the neuron regeneration. Therefore further in-depth investigations are desired to be conducted in order to fully figure out whether DPSCs are able to replace lost cells through their differentiating capacity into mature neurons/oligodendrocytes. Perhaps the alternative explanation could be that DPSCs could release growth factors which induce the local neural stem cells migration and differentiation to replace the lost neurons/oligodendrocytes.

(3) Developing a safer and more reliable system to depositand deliver oral stem/progenitor cells, which also closely relates to the discussion above. Grafted cells will be challenged by the adverse endogenous environment post transplantation which would trigger auto-differentiation of the cells into non-therapeutic cells; therefore it is vitally important to provide a smart delivery system ideally with the capacity of conjugate favourable biomedical factors essential to maintain the multipotency of the grafted cells. Besides, for target specific replacement therapy stem/progenitor cells cannot be delivered in single cell suspension, otherwise the grafted cells will be either lost quickly or diffused without control, therefore a 3D niche of porous structure which constrains the grafted cells would be desirable. From these point of views, nanomaterials based 3D scaffold has been proposed for many years to serve these targets, by rational design of nanomaterials based biocompatible/biodegradable 3D scaffolds with the binding capacity of multiple biological factors essential for multipotency and differentiation. Despite many successful studies showcased the huge potential of such funtionalised nanoscaffold in treating multiple cell types based replacement therapy, such application with oral stem/ progenitor cells is still yet to be explored further.

(4) The microenvironment of transplantion sites should be analyzed more closely because the interaction between the transplanted stem/progenitor cells and endogenous cells may play a critical role in determining the fate of the grafted cells and consequently the outcome of transplantation. Ex-vivo models of stem/progenitor cells transplantation is very useful to disclose the potential interactions between grafted cells vs. endogenous ones, however these can never fully mimic the genuine in-vivo situation where multiple biological factors (both positive and negative) are secreted as part of the pathological response. Therefore such microenvironmental interactions would need to be dealt with using the in-vivo transplantation models, which is inevitably time consuming for the screening of multiple factors regulating the cellular interactions. System biology approach is another very useful tool in the exploration of such intercellular reactions 
involving multiple factors, which is largely neglected and requires more attention in our research community.

(5) The advantage of immunomodulatory properties of oral stem/ progenitor cells deserves more attention in the stem cell replacement therapy. Studies have demonstrated that some of the oral stem / progenitor cells (OMLP-PCs) have no inherent immunogenicity [43], therefore making OMLP-PCs one of the desirable stem cell sources in cell transplantation therapy for reduced immune rejection response. However such suppressed immune responses of the recipient should be fully characterized before clinical applications, and further investigation should be conducted in order to systematically explore the critical roles of oral stem cells in immunomodulation, as well as the interactions between orals stem cells and the immune system.

(6) The mechanism for the regulation of oral stem cells differentiation, proliferation and generation of specific tissue should be fully investigated both in-vitro and in-vivo. More investigation should be performed on the expression of particular genes and sequential activation of associated signaling pathways in the control of such cellular response. The outcome of such studies can be combined with the approaches mentioned above to promote the oral stem cells based regeneration into the desired tissue/cell types with proper functions both in-vitro and in-vivo.

\section{References}

1. Doetschman TC, Eistetter H, Katz M, Schmidt W, Kemler R (1985) The in vitro development of blastocyst-derived embryonic stem cell lines: formation of visceral yolk sac, blood islands and myocardium. J Embryol Exp Morphol 87: $27-45$.

2. Odorico JS, Kaufman DS, Thomson JA (2001) Multilineage differentiation from human embryonic stem cell lines. Stem Cells 19: 193-204.

3. Aufderheide E, Chiquet-Ehrismann R, Ekblom P (1987) Epithelial-mesenchymal interactions in the developing kidney lead to expression of tenascin in the mesenchyme. J Cell Biol 105: 599-608.

4. Vainio S, Karavanova I, Jowett A, Thesleff I (1993) Identification of BMP-4 as a signal mediating secondary induction between epithelial and mesenchymal tissues during early tooth development. Cell 75: 45-58.

5. Kørbling M, Estrov Z (2003) Adult stem cells for tissue repair - a new therapeutic concept? N Engl J Med 349: 570-582.

6. Pittenger MF, Mackay AM, Beck SC, Jaiswal RK, Douglas R, et al. (1999) Multilineage potential of adult human mesenchymal stem cells. Science 284 143-147.

7. Gronthos S, Mankani M, Brahim J, Robey PG, Shi S (2000) Postnatal human dental pulp stem cells (DPSCs) in vitro and in vivo. Proc Natl Acad Sci U S A 97: 13625-13630.

8. Strem BM, Hicok KC, Zhu M, Wulur I, Alfonso Z, et al. (2005) Multipotential differentiation of adipose tissue-derived stem cells. Keio J Med 54: 132-141.

9. Joannides A, Gaughwin P, Schwiening C, Majed H, Sterling J, et al. (2004) Efficient generation of neural precursors from adult human skin: astrocytes promote neurogenesis from skin-derived stem cells. Lancet 364: 172-178.

10. Bieback K, Kern S, Klüter H, Eichler H (2004) Critical parameters for the isolation of mesenchymal stem cells from umbilical cord blood. Stem Cells 22 $625-634$

11. Miura M, Gronthos S, Zhao M, Lu B, Fisher LW, et al. (2003) SHED: stem cells from human exfoliated deciduous teeth. Proc Natl Acad Sci U S A 100 : 5807-5812.

12. Sonoyama W, Liu Y, Fang D, Yamaza T, Seo BM, et al. (2006) Mesenchymal stem cell-mediated functional tooth regeneration in swine. PLoS One 1: e79.

13. Seo BM, Miura M, Gronthos S, Bartold PM, Batouli S, et al. (2004) Investigation of multipotent postnatal stem cells from human periodontal ligament. Lancet 364: 149-155.

14. Angelova Volponi A, Kawasaki M, Sharpe PT (2013) Adult human gingival epithelial cells as a source for whole-tooth bioengineering. J Dent Res 92: 329334

15. Davies LC, Locke M, Webb RD, Roberts JT, Langley M, et al. (2010) A multipotent neural crest-derived progenitor cell population is resident within the oral mucosa lamina propria. Stem Cells Dev 19: 819-830.

16. d'Aquino R, Graziano A, Sampaolesi M, Laino G, Pirozzi G, et al. (2007) Human postnatal dental pulp cells co-differentiate into osteoblasts and endotheliocytes: a pivotal synergy leading to adult bone tissue formation. Cell Death Differ 14 1162-1171.

17. Woods EJ, Perry BC, Hockema JJ, Larson L, Zhou D, et al. (2009) Optimized cryopreservation method for human dental pulp-derived stem cells and their tissues of origin for banking and clinical use. Cryobiology 59: 150-157.

18. Pierdomenico L, Bonsi L, Calvitti M, Rondelli D, Arpinati M, et al. (2005) Multipotent mesenchymal stem cells with immunosuppressive activity can be easily isolated from dental pulp. Transplantation 80: 836-842.

19. Demircan PC, Sariboyaci AE, Unal ZS, Gacar G, Subasi C, et al. (2011) Immunoregulatory effects of human dental pulp-derived stem cells on $T$ cells: comparison of transwell co-culture and mixed lymphocyte reaction systems. Cytotherapy 13: 1205-1220.

20. Zhao Y, Wang L, Jin Y, Shi S (2012) Fas ligand regulates the immunomodulatory properties of dental pulp stem cells. J Dent Res 91: 948-954.

21. Kerkis I, Ambrosio CE, Kerkis A, Martins DS, Zucconi E, et al. (2008) Early transplantation of human immature dental pulp stem cells from baby teeth to golden retriever muscular dystrophy (GRMD) dogs: Local or systemic? J Trans Med 6: 35

22. Patel M, Smith AJ, Sloan AJ, Smith G, Cooper PR (2009) Phenotype and behaviour of dental pulp cells during expansion culture. Arch Oral Biol 54: 898908.

23. Gronthos S, Brahim J, Li W, Fisher LW, Cherman N, et al. (2002) Stem cell properties of human dental pulp stem cells. J Dent Res 81: 531-535.

24. Govindasamy V, Abdullah AN, Ronald VS, Musa S, Ab Aziz ZA, et al. (2010) Inherent differential propensity of dental pulp stem cells derived from human deciduous and permanent teeth. J Endod 36: 1504-1515.

25. Karaoz E, Demircan PC, Saglam O, Aksoy A, Kaymaz F (2011) Human dental pulp stem cells demonstrate better neural and epithelial stem cell properties than bone marrow-derived mesenchymal stem cells. Histochem Cell Biol 136 455-473.

26. Iohara K, Zheng L, Wake H, Ito M, Nabekura J, et al. (2008) A novel stem cell source for vasculogenesis in ischemia: subfraction of side population cells from dental pulp. Stem Cells 26: 2408-2418.

27. Nakatsuka R, Nozaki T, Uemura Y, Matsuoka Y, Sasaki Y, et al. (2010) 5-Aza2'-deoxycytidine treatment induces skeletal myogenic differentiation of mouse dental pulp stem cells. Arch Oral Biol 55: 350-357.

28. Ishkitiev N, Yaegaki K, Imai T, Tanaka T, Nakahara T, et al. (2012) Highpurity hepatic lineage differentiated from dental pulp stem cells in serum-free medium. J Endod 38: 475-480.

29. Paino F, Ricci G, De Rosa A, D'Aquino R, Laino L, et al. (2010) Ectomesenchymal stem cells from dental pulp are committed to differentiate into active melanocytes. Eur Cell Mater 20: 295-305.

30. de Mendonca Costa A, Bueno DF, Martins MT, Kerkis I, Kerkis A, et al. (2008) Reconstruction of large cranial defects in nonimmunosuppressed experimental design with human dental pulp stem cells. J Craniofac Surg 19: 204-210.

31. Riccio M, Maraldi T, Pisciotta A, La Sala GB, Ferrari A, et al. (2012) Fibroin scaffold repairs critical-size bone defects in vivo supported by human amniotic fluid and dental pulp stem cells. Tissue Eng Part A 18: 1006-1013.

32. Zheng Y, Liu Y, Zhang CM, Zhang HY, Li WH, et al. (2009) Stem cells from deciduous tooth repair mandibular defect in swine. J Dent Res 88: 249-254.

33. Yamada $Y$, Ito K, Nakamura S, Ueda M, Nagasaka T (2011) Promising cellbased therapy for bone regeneration using stem cells from deciduous teeth, dental pulp, and bone marrow. Cell Transplant 20: 1003-1013.

34. Ito K, Yamada Y, Nakamura S, Ueda M (2011) Osteogenic potential of effective bone engineering using dental pulp stem cells, bone marrow stem cells, and periosteal cells for osseointegration of dental implants. Int $\mathrm{J}$ Oral Maxillofac Implants 26: 947-954. 
Citation: Jiang W, Ni L, Sloan A, Song B (2015) Tissue Engineering and Regenerative Medicine, From and Beyond the Dentistry. Dentistry 5: 306. doi:10.4172/2161-1122.1000306

35. Sasaki R, Aoki S, Yamato M, Uchiyama H, Wada K, et al. (2008) Tubulation with dental pulp cells promotes facial nerve regeneration in rats. Tissue Eng Part A 14: 1141-1147.

36. Arthur A, Shi S, Zannettino AC, Fujii N, Gronthos S, et al. (2009) Implanted adult human dental pulp stem cells induce endogenous axon guidance. Stem Cells 27: 2229-2237.

37. Apel C, Forlenza OV, de Paula VJ, Talib LL, Denecke B, et al. (2009) The neuroprotective effect of dental pulp cells in models of Alzheimer's and Parkinson's disease. J Neural Transm 116: 71-78.

38. Nesti C, Pardini C, Barachini S, D'Alessandro D, Siciliano G, et al. (2011) Human dental pulp stem cells protect mouse dopaminergic neurons against MPP+ or rotenone. Brain Res 1367: 94-102.

39. Sakai K, Yamamoto A, Matsubara K, Nakamura S, Naruse M, et al. (2012) Human dental pulp-derived stem cells promote locomotor recovery after complete transection of the rat spinal cord by multiple neuro-regenerative mechanisms. J Clin Invest 122: 80-90.

40. Gandia C, Arminan A, Garcia-Verdugo JM, Lledo E, Ruiz A, et al. (2008) Human dental pulp stem cells improve left ventricular function, induce angiogenesis, and reduce infarct size in rats with acute myocardial infarction. Stem Cells 26 : 638-645

41. Nishino Y, Ebisawa K, Yamada Y, Okabe K, Kamei Y, et al. (2011) Human deciduous teeth dental pulp cells with basic fibroblast growth factor enhance wound healing of skin defect. J Craniofac Surg 22: 438-442.

42. Stephens P, Genever P (2007) Non-epithelial oral mucosal progenitor cell populations. See comment in PubMed Commons below Oral Dis 13: 1-10.

43. Davies LC, Lönnies H, Locke M, Sundberg B, Rosendahl K, et al. (2012)
Oral mucosal progenitor cells are potently immunosuppressive in a doseindependent manner. See comment in PubMed Commons below Stem Cells Dev 21: 1478-1487.

44. Arthur A, Rychkov G, Shi S, Koblar SA, Gronthos S (2008) Adult human dental pulp stem cells differentiate toward functionally active neurons under appropriate environmental cues. Stem Cells 26: 1787-1795.

45. Chang CC, Chang KC, Tsai SJ, Chang HH, Lin CP (2014) Neurogenic differentiation of dental pulp stem cells to neuron-like cells in dopaminergic and motor neuronal inductive media. J Formos Med Assoc 113: 956-965.

46. Gervois P, Struys T, Hilkens P, Bronckaers A, Ratajczak J, et al. (2014) Neurogenic maturation of human dental pulp stem cells following neurosphere generation induces morphological and electrophysiological characteristics of functional neurons. Stem Cells Dev 24: 296-311.

47. Iohara K, Zheng L, Ito M, Tomokiyo A, Matsushita K, et al. (2006) Side population cells isolated from porcine dental pulp tissue with self-renewal and multipotency for dentinogenesis, chondrogenesis, adipogenesis, and neurogenesis. Stem Cells 24: 2493-2503.

48. Osathanon T, Nowwarote N, Pavasant $P$ (2011) Basic fibroblast growth factor inhibits mineralization but induces neuronal differentiation by human dental pulp stem cells through a FGFR and PLCgamma signaling pathway. J Cell Biochem 112: 1807-1816.

49. Sasaki R, Aoki S, Yamato M, Uchiyama H, Wada K, et al. (2008) Neurosphere generation from dental pulp of adult rat incisor. Eur J Neurosci 27: 538-548.

50. Király M, Kádár K, Horváthy DB, Nardai P, Rácz GZ, et al. (2011) Integration of neuronally predifferentiated human dental pulp stem cells into rat brain in vivo. Neurochem Int 59: 371-381. 